\title{
Multiple sclerosis in Finland 2018-Data from the national register
}

\section{Laakso, Sini M.}

2019-11

Laakso, S M , Viitala , M , Kuusisto , H, Sarasoja , T, Hartikainen , P , Atula , S , Tienari , P $\mathrm{J} \&$ Soilu-Hänninen , M 2019 , ' Multiple sclerosis in Finland 2018-Data from the national register ' , Acta Neurologica Scandinavica , vol. 140 , no. 5 , pp. 303-311 . https://doi.org/10.1111/ane.13145

http://hdl.handle.net/10138/312954

https://doi.org/10.1111/ane.13145

acceptedVersion

Downloaded from Helda, University of Helsinki institutional repository.

This is an electronic reprint of the original article.

This reprint may differ from the original in pagination and typographic detail.

Please cite the original version. 
DR. SINI M LAAKSO (Orcid ID : 0000-0002-0104-2849)

Article type : Original Article

\title{
Multiple sclerosis in Finland 2018- data from the national register
}

Sini M Laakso, MD PhD ${ }^{1}$, Matias Viitala, $\mathrm{MSc}^{2}$, Hanna Kuusisto, $\mathrm{MD} \mathrm{PhD}^{3}$, Taneli Sarasoja, $\mathrm{MD}^{4}$, Päivi Hartikainen, MD, $\mathrm{PhD}^{5}$, Sari Atula, $\mathrm{MD} \mathrm{PhD}^{1}$, Pentti J. Tienari, $\mathrm{MD} \mathrm{PhD}^{1}$, Merja Soilu-Hänninen, $\mathrm{MD} \mathrm{PhD}^{6}$

${ }^{1}$ Helsinki University Hospital, Neurocenter, and University of Helsinki, Department of Neurosciences, Helsinki, Finland, ${ }^{2}$ University of Turku, Department of Mathematics and Statistics, and StellarQ Ltd, Turku, Finland, ${ }^{3}$ Tampere University Hospital, Department of Neurology, Tampere, Finland, and University of Eastern Finland, Department of Health and Social management, Kuopio, Finland, ${ }^{4}$ Central Hospital of Central Finland, Department of Neurology, Jyväskylä, Finland, ${ }^{5}$ Kuopio University Hospital, Department of Neurology, and University of Eastern Finland, Department of Neurology, Kuopio, Finland, ${ }^{6}$ Turku University Hospital, Division of Clinical Neurosciences, and University of Turku, Department of Neurology, Turku, Finland

Corresponding author: Sini M Laakso, MD PhD

Neurocenter, Helsinki University Hospital, PB 372, 00029 HUS, Helsinki, Finland

E-mail: sini.m.laakso@hus.fi

Tel: +358-9-471 72261

\section{Acknowledgements}

The authors gratefully acknowledge the contribution of the StellarQ IT team for building and continuously developing the Finnish MS Register IT platform. All members of the Steering committee of the Finnish MS register are gratefully acknowledged. Building up the Finnish MS register has received funding from Biogen Idec, Merck, Novartis, Sanofi Genzyme, Roche, Teva and Business Finland.

\begin{abstract}
Objectives: Finland is a high-risk multiple sclerosis (MS) region, but a national MS register has not existed until 2014. In this paper we present the Finnish MS register variables and data collected by 31.12.2018.
\end{abstract}

This article has been accepted for publication and undergone full peer review but has not been through the copyediting, typesetting, pagination and proofreading process, which may lead to differences between this version and the Version of Record. Please cite this article as doi: 10.1111/ane.13145

This article is protected by copyright. All rights reserved. 
Materials \& Methods: Numbers and data counts of MS patients in the register (ICD-10 code G35) are presented. The disease types and proportion of patients receiving disease modifying treatments (DMTs) were analyzed in five hospital districts with most complete data sets. MS prevalence in Finland was estimated using administrative hospital discharge data as an additional resource.

Results: There were a total of 8722 MS patients in the Finnish MS register by 31.12.2018 (71.5\% females). Mean age at MS diagnosis was 38.7 years and peak prevalence was at age 50-54 years. Disease course was relapsing remitting (RRMS) in $66.7 \%$, secondary progressive (SPMS) in $13.5 \%$, and primary progressive (PPMS) in $7.9 \%$ of the 5365 MS patients in the selected districts with most complete data. A total of $66.0 \%$ of RRMS patients, $19.6 \%$ of SPMS patients and $9.9 \%$ of PPMS patients were receiving DMTs. By combining MS register data with databases of those hospitals that had not joined the register, the nation-wide prevalence estimate was between 10 and 11 thousand patients (corresponding to crude prevalence 180-200/100,000).

Conclusions: The Finnish MS register is currently used in 15/21 Finnish hospital districts. By register integration into the electronic patient files, the coverage of the register has increased to approximately $80 \%$ of the estimated Finnish MS population.

\section{Keywords}

Multiple Sclerosis; Electronic Health Records; Treatment Outcome, Registry, Prevalence

\section{Introduction}

Finland is a high-risk multiple sclerosis (MS) region based on regional studies (1-3), but nationwide MS prevalence studies have not been performed recently. A national MS register was launched in 2014, which enables systematic follow-up of incidence and prevalence figures as well as many clinical variables. Disease modifying treatments (DMTs) for MS have developed rapidly during the last decade. Therefore structured documentation of the complex treatment profiles of individual patients is becoming increasingly important. The DMTs are considered more effective when administered early on during the disease course (4). Therefore, predictive tools to identify patients with highly active disease, who receive greatest benefit from the most effective therapies early on, are needed. Moreover, there is a need for a tool to better assess the efficacy, safety and cost - benefit ratio of the emerging new DMTs at the national level.

MS registers attempt to bridge these gaps. They are in active use in many European countries (5), and the need to further develop these registers is recognized worldwide (6). Registers assist the clinician as a decision-making tool in individualized patient follow-up by providing a graphical presentation of the patient's entire disease history. Registers can provide real-world data on the effectiveness, tolerability and safety of MS drugs (7). The Norwegian MS register connects with biobank information, thereby facilitating research on biomarkers for MS (8). A prediction tool to predict the individual's risk of conversion to secondary progressive multiple sclerosis (SPMS) at the time of MS onset has been recently published based on data derived from one of the most successful national MS registers, the Swedish MS register (9).

This article is protected by copyright. All rights reserved. 
The Finnish MS register is a browser-based quality register for public health care organizations. The software service is provided by StellarQ (stellarq.com). The development of the register, the steering committee and the core features of the register are described at the register home page (neurorekisteri.fi). By 2018, 15/21 of Finland's public hospital districts including all five university hospitals and ten central hospitals have joined the service, and most of them have integrated it into their electronic patient documentation system. Statistics of realtime aggregated anonymized national level data are available to all hospitals who have joined the register. At the moment the register is not covering all MS patients in Finland, but an effort is ongoing to achieve complete coverage. The funding of the register is based on licensing fees paid by Finnish hospital districts to the software service provider.

The objective of this study is to describe the characteristics and status of launching of the Finnish MS register. We illustrate the register core variables and the data collected. We provide a rough estimate of the prevalence of MS in Finland, and show patient demographics from five hospital districts with more detailed register data. We discuss the challenges and future perspectives of this new national MS register.

\section{Materials and Methods}

The national level data was collected from the real-time aggregated anonymized portal of the MS register. Data entries into the register were performed either manually by the treating neurologist or by diagnosis integration from the hospital administrative data. In both cases, the validity of the G35 and G37.9 diagnoses was verified by chart review. A more detailed analysis of the register data was carried out from five hospital districts: Helsinki and Uusimaa, Southwest Finland, Tavastia Proper, Northern Savonia and Central Finland. Rationale for this selection was that these five hospital districts have entered the patient data both retrospectively and prospectively. Prospective data collection started during 2014-2016. Retrospective disease history data from the disease onset for each patient has been filled in manually by the treating neurologists, residents, MS nurses, or medical students under supervision of the MS register steering committee members. Data from these five hospital districts was collected separately from each hospital's closed register, anonymized and then pooled for statistical analysis.

Following data cleansing was used to derive datasets ready for statistical analysis: partial dates were imputed as first day of the month or first day of the year depending on the imputation needs, duplicated records were removed, and possible discrepant medication events were corrected to be logical. Mean, standard deviation, and frequencies were used to produce patient demographics.

This study was approved by the institutional review boards of all the participating hospitals. According to the Finnish law, approval of ethical committee was not required, as the study was based on administrative register data and included no contact with patients. Office of Data Protection in Finland and the Ministry of Social Affairs and Health have endorsed their statements, that no informed consent is needed for entering the patient data into the Finnish MS register, since it is regarded as a part of the hospital's patient documentation system. Each hospital district is a register keeper for their own data.

This article is protected by copyright. All rights reserved. 


\section{Results}

\section{MS register coverage in the Finnish health care districts}

The Finnish MS register was developed foremost to serve the clinician in the daily practice when evaluating treatment choices for an MS patient in the outpatient clinic. Therefore, a strong emphasis was given to the graphical design of the user interface. Figure 1 shows the front page of the user interface, where the key variables of diagnosis date and disease type, current and past EDSS scores and treatment and relapse histories are visualized graphically.

Finland is divided into 21 public health care districts, with very different population sizes. The decentralized model of health care has been a challenge to the MS register launching, since the register use is voluntary and each hospital has decided individually whether to join the register or not. Figure 2 shows the health care districts in Finland and which of them have joined the register by 31.12.2018.

At the national level, there were a total of 8722 MS patients (G35) and 512 patients with unspecified demyelinative disease of the central nervous system (G37.9) registered in the MS register by $31.12 .2018(71.5 \%$ females). Mean and median ages at MS diagnosis were 38.7 and 37.0 years, respectively, and peak prevalence was at age 50-54 years. The number of G35 patients obtained from the hospital administrative databases from the hospitals not yet joined was 778 (Figure 2, discharge data). The number of patients not registered in the hospital districts that are using manual entry of patients (Figure 2, nine districts), were identified from the hospital administrative databases. This procedure identified 1400 additional patients. These three data sets in combination provide an estimate of MS prevalence in Finland up to 10900 at December 31, 2018. No medical record review was performed to the patients identified from the administrative databases, a single entry of diagnosis G35 was enough for inclusion, and patients in both in- and outpatient care were included. The number of MS patients with reimbursement rights for DMTs of MS (Social Insurance Institution of Finland, insurance code 109 for G35) was 9372 at 31.12.2018 (11). Since all patients are not applied for the reimbursement rights for the DMTs of MS, this figure does not capture all of the Finnish MS patients. Those MS patients who receive infusion therapies, paid entirely from the hospital budgets, may not be captured based on the reimbursement rights. According to our register data, approximately $7 \%$ of the patients received one of the infusion therapies as a first line therapy.

To provide the structure and comprehensiveness of the data collected by the Finnish MS register, we present the register variables and the data counts of each variable included in the register. Table 1 describes the variables, how many times the variable has been entered in the register, and which proportion of the patients have the information included in their data file.

\section{Demographics and disease characteristics of MS patients in the hospital districts with retrospective and prospective data}

At the national level, only $62.9 \%$ of patients had their relapses entered into the register (Table 1 ). Therefore, we limited our more detailed analysis into those hospital districts where both retrospective and prospective patient disease history data had been entered into their register. We performed the more detailed analysis of register data from the following five hospital districts: Helsinki and Uusimaa, Southwest Finland, Tavastia Proper, Northern Savonia and Central Finland (Figure 2 dark blue regions). In Table 2 we present the demographics and disease characteristics of MS patients from the five hospital districts. In this data-set there were $5365 \mathrm{MS}$ patients, $66.7 \%$ had RRMS, $13.5 \%$ SPMS and 7.9\% PPMS. A total of $5.7 \%$ of the RRMS patients were

This article is protected by copyright. All rights reserved. 
classified as having a highly active MS defined by at least two relapses during the previous year or at least one relapse during the previous year in spite of DMT treatment. A total of $66.0 \%$ of RRMS patients were currently receiving a DMT, and $16.6 \%$ of the RRMS patients were treated with fingolimod, cladribine, natalizumab, ocrelizumab, rituximab, mitoxantrone or alemtuzumab, which were considered as escalation treatments or treatment choices for highly active MS according to the national MS treatment guidelines (16). A total of 6.7\% of the patients were receiving infusion therapies as the first line treatment. The most used DMT options in the PPMS patients were rituximab and azathioprine (data not shown).

The mean age of a patient with RRMS in the five hospitals cohort of MS patients was 48.2 years and in patients with SPMS 61.7 years (Table 2). The age distribution of the patients is shown in Figure 3A. The age of 52.0 years, disease duration over 16.5 years and EDSS 3.5 seemed to determine a cut-off point between RRMS and SPMS disease stages (Figure 3A, 3B and 3C).

\section{Discussion}

A key feature of the Finnish MS register is the possibility for integration of the register core variables directly into the electronic patient documentation system of each participating hospital. This enables automated transfer of new MS diagnoses and all other integrated register variables into the register as well as automated updates of the deceased patients. In Finland, MS is exclusively diagnosed and mostly treated by neurologists working in public health care. Once the register use and the diagnosis integrations cover all the public hospitals, the register can provide a full coverage and a constant update on the national incidence and prevalence of MS. The Finnish MS register coverage for the core variables of patient demographics and date/year of diagnosis has risen to cover approximately $80 \%$ of the MS patients within 5 years after its launch. Both retrospective and prospective disease history information is currently available from approximately half of the patients, but it has to be taken into consideration that the retrospective disease history data has been entered in some hospitals also by medical students and MS nurses and not solely by the treating neurologists. This could influence the reliability of the retrospective data.

Based on published regional figures from 1990's, estimates of the prevalence of MS in Finland have been approximately 7000 in a population of 5.5 million $(17,18)$. The numbers of MS patients alive with reimbursement rights for DMTs of MS (Social Insurance Institution of Finland, insurance code 109 for G35) was 9027 by the end of 2017 and 9372 at 31.12.2018 (11). Since all patients do not apply for the reimbursement rights for the DMTs, this figure does not capture all of the Finnish MS patients. In our recent paper investigating the prevalence of MS in Southwest Finland and North Carelia hospital districts, we showed standardized prevalence figures of 280 and 168 per 100000 , respectively, in the age group over 10 years ( 4.9 of the 5.5 million inhabitants). This suggests a longitudinal gradient and yields a combined prevalence of 247/100 000, which would give a total number of approx. 13500 patients applied to the total population. Previously, a much smaller crude prevalence of 103/ 100000 in Northern Finland has been published (19) suggesting a latitudinal gradient and supporting a smaller national prevalence than estimated by the combined prevalence of Western and Eastern Finland. There were large regional differences in the estimated prevalence in our study consistent with the previously published data, but due to different ways of data capture between hospital districts (administrational data vs register data), firm conclusions of longitudinal and latitudinal prevalence gradients in our country remain to be established in further studies.

This article is protected by copyright. All rights reserved. 
Based on this and the data presented in the current paper, we estimate that the number of MS patients in our country is between 10 and 11000 patients in a population of 5.5 million. Some of the registered 512 patients with G37.9 diagnosis (mostly clinically isolated syndrome, CIS) could possibly fulfill the 2017 McDonald diagnostic criteria for MS if they were re-evaluated, but their number is small and does not materially change our estimate of MS prevalence in Finland at the end of year 2018. The rise in the MS prevalence in our country reflects the globally acknowledged increasing trend of MS prevalence, attributed to more sensitive diagnostics, improved prognosis and increasing female to male-ratio with higher life expectancy in females $(20,21)$. The female-to-male ratio in our national registry cohort was 2.5 at 31.12.2018, while ratios ranging from 1.6 to 2.4 in different regions in Finland have been published from a period spanning from 1979 to 1993 (18). A more striking increase has occurred in the mean age of the patients from 38.2 years between years 1979 to 1993 (18) to 51 years in the current registry cohort. This suggests that aging of the population and improved survival has a more pronounced role in the increasing prevalence than a changing gender ratio.

The natural tendency of RRMS to transform into SPMS in patients without DMTs used is considerable. In older studies, 58.2\% reach SPMS within a median of 18.9 years (22). In a more recent study from the treatment era, only $18.1 \%$ of RRMS patients confronted this conversion after a median of 16.8 years (23). Our data contain both patients diagnosed before and after the DMT era with varying treatment histories, and therefore straight comparisons to either of these results cannot be made. In the light of EDSS accumulation, it has been reported that after EDSS 3 there is a tendency of both treated and treatment-naive MS to transform into SPMS, and present thereafter with steady incline in disability (24). The EDSS distributions of our RRMS and SPMS patient cohorts are in line with this finding, because after reaching EDSS 3.5 the proportion of RRMS patients sharply declined (Figure 3).

The proportion of Finnish RRMS patients currently treated with DMT's was $66.0 \%$, which is similar to that previously reported from Sweden (25). The proportion of SPMS patients is likely to be underreported and may reflect the reluctance of clinicians to identify the patients as having secondary progressive MS because of lacking effective DMT options for this disease phase. The proportion of SPMS patients treated with DMTs was $19.6 \%$ in our analysis. Additionally, $9.9 \%$ of the patients with PPMS were also receiving DMTs although the first DMT licensed to PPMS did not become available in Finland until February 2018.

There is a subgroup of RRMS patients who have a more aggressive disease course called highly active MS that has been estimated to comprise about $4-15 \%$ of the patients (26). There is no consensus criteria or treatment algorithms for these patients, but MRI lesions that enhance Gadolinium despite treatment, an EDSS scale of 4 points at 5 years of onset of the disease, no response to treatment with one or more DMTs for at least one year and multiple relapses (two or more) with incomplete recovery in the ongoing year, belong to the criteria. Our data from the five hospital districts showed a prevalence of $5.7 \%$ of MS patients with highly active disease defined by at least two relapses during the previous year or at least one relapse during the previous year in spite of DMT treatment. Adding the MRI and EDSS criteria will allow better identification of these patients in the future.

Now five years after its launch, the Finnish MS register serves the majority of Finnish neurologists taking care of MS patients and is increasingly adopted as a part of clinical practice. It has clear benefits regarding the concise presentation of all clinical data for an MS patient, allowing the clinician to easily get an overview of the patient's disease course and medications instead of browsing through a large quantity of medical records. Therefore, it is easier to make quicker data-based treatment decisions during the patient visit. The graphic view of the course of the disease can be shown to the patient at the visit, enabling the patient to take part in the

This article is protected by copyright. All rights reserved. 
decision making when for instance initiating or changing the treatment. It also helps the patients to understand the grounds for determining the type of the disease and disease activity. MS register is an excellent means to empower patients to take charge of their disease and commit to the treatment, rehabilitation and beneficial life style choices. Also, during the partially virtual expert consultations for more challenging patients, all participants get a quick digital picture of the patient history, based on which to make treatment decisions.

The challenges lie in broadening the scope of data entries to include also e.g. MRI results, and to ensure the comprehensive longitudinal data collection of existing variables. Changes in electronic patient medical record systems require constant updates of the integrations. Use of the register is not compulsory and funding is based on the decision of each hospital district to include the register fees into their tightening budgets. Funding has raised concern also in other European countries (5), and MS registers need to continuously prove their benefits.

As the register becomes more detailed and covers all MS patients, research questions regarding both MS epidemiology and real-world data can be more efficiently addressed. Subgroups such as patients with highly active MS can be identified from the MS register, facilitating targeted patient recruitment for clinical trials or other research purposes. Genome-wide single nucleotide polymorphism data is generated in Finland through the national Finngen project (finngen.fi) from samples donated to biobanks, covering whole Finland (finbb.fi). The genetic data is made available for researchers and these data in combination with the Finnish MS register's clinical information make efficient pharmacogenomic analyses possible on a nation-wide scale. Moreover, associations of many clinical parameters (e.g. disease course, prognosis, co-morbidity) with genetic markers can be tested in an efficient manner and eventually it will be possible to recruit patients to clinical trials on the basis of demographic, clinical and genetic variables.

\section{Conflict of interest}

S M L: Fee for lecture Merck; congress expenses Roche, Merck

M V: No disclosures

H K: Fees for lectures Teva, Novartis, Sanofi Genzyme, Biogen, Merck; congress expenses Zamboni, Teva, Merck, Sanofi Genzyme; advisory boards Teva, Roche, Novartis, Sanofi Genzyme, Biogen, Merck.

P H: Research funding Biogen, Sanofi Genzyme; congress expenses Sanofi Genzyme, Merck, Teva, Allergan, Roche, NordicInfu Care, Biogen; fees for lectures and advisory boards Sanofi Genzyme, Merck, Teva, Allergan, Roche, NordicInfu Care, Biogen.

T S: Congress expenses Biogen, Novartis, Teva, Roche.

S A: Fees for lectures Merck, Roche, Santen; congress expenses Biogen, Sanofi Genzyme, Merck, Orion, Pfizer; advisory boards Biogen, Sanofi Genzyme, Merck, Pfizer, Roche.

P J T: Research grants Biogen, Merck, Novartis, Sanofi-Genzyme, Roche; fees for lectures Merck, Roche, Santen, Orion, Biogen, Sanofi Genzyme, Novartis, Pfizer, Teva; congress expenses Biogen, Sanofi Genzyme, Merck, Novartis, Bayer, Teva.

This article is protected by copyright. All rights reserved. 
M S-H: Fees for lectures Biogen, Novartis, Merck, Roche, Sanofi Genzyme, Teva; congress expenses Biogen, Merck, Roche, Sanofi Genzyme, Teva; advisory boards Biogen, Novartis, Merck, Roche, Sanofi Genzyme, Teva.

\section{Data Availability Statement}

The data that support the findings of this study are available from the corresponding author upon reasonable request.

This article is protected by copyright. All rights reserved. 


\section{References}

1. Åivo J, Kurki S, Sumelahti ML, Hänninen K, et al. Risk of osteoporotic fractures in multiple sclerosis patients in southwest Finland. Acta Neurol Scand 2017; 135:516-521.

2. Pirttisalo AL, Soilu-Hänninen M, Sipilä, JOT. Multiple sclerosis epidemiology in Finland: regional differences and high incidence. Acta Neurol Scand 2019; 139(4):353-359.

3. Holmberg M, Murtonen A, Elovaara I, Sumelahti ML. Increased female MS incidence and differences in gender-specific risk in medium- and high-risk regions in Finland from 1981-2010. Mult Scler Int 2013; 2013:182516

4. Trojano M, Tintore M, Montalban X, Hillert J, et al. Treatment decisions in multiple sclerosis - insights from real-world observational studies. Nat Rev Neurol 2017; 13(2):105-118.

5. Glaser A, Stahmann A, Meissner T, Flachenecker P, et al. Multiple sclerosis registries in Europe - An updated mapping survey. Mult Scler Relat Disord 2018; 27:171-178.

6. Bebo BF Jr, Fox RJ, Lee K, Utz U, et al. Landscape of MS patient cohorts and registries: recommendations for maximizing impact. Mult. Scler. 2018; 24(5):579-586.

7. Ziemssen T, Hillert J, Butzkueven $\mathrm{H}$. The importance of collecting structured clinical information on multiple sclerosis. BMC Med 2016; 14:81.

8. Myhr KM, Grytten N, Torkildsen $\emptyset$, Wergeland S, et al. The Norwegian Multiple Sclerosis Registry and Biobank. Acta Neurol Scand 2015; 132(199):24-8.

9. Manouchehrinia A, Zhu F, Piani-Meier D, Lange M, et al. Predicting risk of secondary progression in multiple sclerosis: A nomogram. Mult Scler 2018; Jun 1:1352458518783667.

10. Tilastokeskus. Väestö sairaanhoitopiireittäin 2018, väestö 31.12.2017. [pxnet2.stat.fi/PXWeb/pxweb/fi/StatFin/], visited 28.12.2018.

11. Social Insurance Institution of Finland. Statistical Yearbook of the Social Insurance Institution 2017. Available online at: https://www.kela.fi/web/en/statistical-publications_statistical-yearbook

12. Smith A. Symbol digit modalities test: manual. Los Angeles CA:Western Physiological Services, 1982.

13. Rosti-Otajärvi E, Hämäläinen $P$, Wiksten $A$, Hakkarainen T, et al. Validity and reliability of the Finnish version of the Multiple Sclerosis Impact Scale-29. Brain Behav 2017; 7(7):e00725.

14. Sintonen H.The 15D instrument of health-related quality of life: properties and applications. Ann Med 2001; $33(5): 328-36$

15. Rosti-Otajärvi E, Hämäläinen $P$, Wiksten A, Hakkarainen T, et al. Validity and reliability of the Fatigue Severity Scale in Finnish multiple sclerosis patients. Brain Behav 2017; 7(7):e00743.

16. Multiple sclerosis. Current Care Guidelines. Working group set up by the Finnish Medical Society Duodecim and the Finnish Neurological Society. Helsinki: The Finnish Medical Society Duodecim, 2015 (referred Jan 8, 2019). Available online at: www.kaypahoito.fi

17. Sumelahti ML, Tienari PJ, Wikstrom J, Palo J, et al. Increasing prevalence of multiple sclerosis in Finland. Acta Neurol Scand 2001; 103(3):153-158.

18. Sumelahti ML, Tienari PJ, Hakama M, Wikström J. Multiple sclerosis in Finland: incidence trends and differences in relapsing remitting and primary progressive disease courses. J Neurol Neurosurg Psychiatry 2003; 74(1):25-8.

This article is protected by copyright. All rights reserved. 
19. Krökki O, Bloigu R, Reunanen M, Remes AM. Increasing incidence of multiple sclerosis in women in Northern Finland. Mult Scler 2011; 17(2):133-8.

20. Koch-Henriksen N, Sørensen PS. The changing demographic pattern of multiple sclerosis epidemiology. Lancet Neurol 2010; 9(5):520-532.

21. Kingwell E, Marriott JJ, Jetté N, Pringsheim T, et al. Incidence and prevalence of multiple sclerosis in Europe: A systematic review. BMC Neurol 2013; 13:128.

22. Tremlett H, Yinshan Z, Devonshire V. Natural history of secondary-progressive multiple sclerosis. Mult Scler 2008; 14(3):314-24.

23. University of California, San Francisco MS-EPIC Team, Cree BA, Gourraud PA, Oksenberg JR, et al. Long-term evolution of multiple sclerosis disability in the treatment era. Ann Neurol. 2016; 80(4):499-510.

24. Leray E, Yaouanq J, Le Page E, Coustans M, et al. Evidence for a two-stage disability progression in multiple sclerosis. Brain 2010; 133(7):1900-1913.

25. Hillert J, Stawiarz L. The Swedish MS registry - clinical support tool and scientific resource. Acta Neurol Scand. 2015; 132:11-19.

26. Díaz C, Zarco LA, Rivera DM. Highly active multiple sclerosis: An update. Mult Scler Relat Disord 2019; 24 (30): 215-224.

This article is protected by copyright. All rights reserved. 
Table 1. Variables included in the Finnish MS register and their data counts

\begin{tabular}{|c|c|c|}
\hline Data sections & Data counts & $\%$ patients with data \\
\hline \multicolumn{3}{|l|}{ Demographics (mandatory) } \\
\hline Name and identification number & 9234 & $9234(100.0)$ \\
\hline Date of birth & 9234 & $9234(100.0)$ \\
\hline Gender & 9234 & $9234(100.0)$ \\
\hline Municipality & 8315 & $8315(90.0)$ \\
\hline \multicolumn{3}{|l|}{ Diagnosis (mandatory) } \\
\hline Date/year of onset of symptoms & 5038 & $5038(54.6)$ \\
\hline Date/year of diagnosis & 8722 & $8722(94.5)$ \\
\hline Diagnosis type (G35,G37.9, conversion to SPMS)* & 11574 & $9234(100.0)$ \\
\hline Clinical course (RRMS, SPMS, PPMS) & 6720 & $5865(63.5)$ \\
\hline Anatomical localization(s) of initial symptoms & 1251 & $1251(13.5)$ \\
\hline MRI at diagnosis (optional) & 1008 & $990(10.7)$ \\
\hline CSF-analyses at diagnosis (optional) & 791 & $775(8.4)$ \\
\hline \multicolumn{3}{|l|}{ Disease modifying treatment (mandatory) } \\
\hline Treatment history (start and end dates) & 7725 & $3658(39.6)$ \\
\hline Current treatment (start date of current treatment) & 3979 & $3979(43.1)$ \\
\hline Adverse events & 2275 & $1448(15.7)$ \\
\hline Reasons for treatment discontinuation & 7230 & $3559(38.5)$ \\
\hline \multicolumn{3}{|l|}{ Relapses } \\
\hline Date & 19585 & $5812(62.9)$ \\
\hline Symptoms & 18668 & $5690(61.6)$ \\
\hline Corticosteroid treatment (mandatory) & 13419 & $4421(47.9)$ \\
\hline \multicolumn{3}{|l|}{ EDSS score (mandatory) } \\
\hline Scores & 14530 & $4035(43.7)$ \\
\hline \multicolumn{3}{|l|}{ MRI (optional) } \\
\hline Date & 13489 & $4588(49.7)$ \\
\hline Total number of $\mathrm{T} 2$ lesions (semiquantitative) & 3199 & $1506(16.3)$ \\
\hline $\begin{array}{l}\text { Number of new lesions or arrow indicating increased } \\
\text { lesion load }\end{array}$ & 1549 & $1001(10.8)$ \\
\hline Gadolinium use & 8338 & $3569(38.7)$ \\
\hline \multicolumn{3}{|l|}{ Symbol Digit Modalities Test result [SDMT (12)] } \\
\hline Scores & 373 & $127(1.4)$ \\
\hline \multicolumn{3}{|l|}{ Lab test results } \\
\hline CSF analysis of cells and IgG production & 2967 & $2620(28.4)$ \\
\hline Blood biomarkers: & & \\
\hline JCV serology & 705 & $431(4.7)$ \\
\hline Serum 25-D concentration & 2664 & $1632(17.7)$ \\
\hline \multicolumn{3}{|l|}{ Patient reported outcome measure MyMS (optional) } \\
\hline Number of patients registered & 477 & $477(5.2)$ \\
\hline Multiple Sclerosis Impact Scale [MSIS-29 (13)] & 213 & $197(2.1)$ \\
\hline Quality of life 15D (14) & 138 & $129(1.4)$ \\
\hline Fatigue severity scale [FSS (15)] & 293 & $252(2.7)$ \\
\hline Comorbidities & 981 & $763(8.3)$ \\
\hline
\end{tabular}

This article is protected by copyright. All rights reserved. 
\begin{tabular}{|l|l|l|} 
Smoking & 1126 & $1022(11.1)$ \\
Education & 574 & $519(5.6)$ \\
Employment & 1083 & $956(10.4)$ \\
Family history of MS & 170 & $160(1.7)$ \\
\hline
\end{tabular}

Footnote to Table 1: 8722 MS (G35) and 512 unspecified demyelinative disease of the central nervous system (G37.9) patients (total of 9234) were registered at 31.12.2018. There can be multiple entries of data for each data section and therefore the data counts differ from the number of patients. Diagnosis type data count exceeds the total number of patients because conversion to SPMS is entered as a new diagnosis.

Table 2. Demographics and disease characteristics of MS patients from the five hospital districts

\begin{tabular}{|c|c|c|c|}
\hline Diagnosis & Number of patients $(\%)$ & Age (Years; Mean, SD) & Gender (\% F/M) \\
\hline G35 & $5365(95.0 \%)$ & $51.7(14.1)$ & $72.4 / 27.6$ \\
\hline G37.9 & $284(5.0 \%)$ & $47.3(13.0)$ & $71.5 / 28.5$ \\
\hline All & $5649(100.0 \%)$ & $51.3(13.6)$ & $72.4 / 27.7$ \\
\hline Disease type & Number of patients $(\%)$ & Age (Years; Mean, SD) & Gender (\% F/M) \\
\hline RRMS & $3578(66.7 \%)$ & $48.2(12.6)$ & $74.5 / 25.5$ \\
\hline SPMS & $723(13.5 \%)$ & $61.7(10.4)$ & $69.0 / 31.0$ \\
\hline PPMS & $425(7.9 \%)$ & $62.3(10.2)$ & $62.1 / 37.9$ \\
\hline $\begin{array}{l}\text { Highly active } \\
\text { disease }\end{array}$ & Number of patients $(\%)$ & Age (Years; Mean, SD) & Gender $(\%$ F/M $)$ \\
\hline RRMS & $195(5.7 \%)$ & $43.4(10.3)$ & $75.4 / 24.6$ \\
\hline SPMS & $17(2.4 \%)$ & $57.9(10.0)$ & $76.5 / 23.5$ \\
\hline $\begin{array}{l}\text { Number of } \\
\text { patients with } \\
\text { DMTs }(\%)\end{array}$ & Any DMT & $\begin{array}{l}\text { IFN, GA, DMF or } \\
\text { TRF }\end{array}$ & $\begin{array}{l}\text { Fingolimod, } \\
\text { cladribine or } \\
\text { infusion therapies }\end{array}$ \\
\hline All & $2730 / 5365(50.9 \%)$ & $1974 / 5365(36.8 \%)$ & $756 / 5365(14.1 \%)$ \\
\hline RRMS & $2363 / 3578(66.0 \%)$ & $1769 / 3578(49.4 \%)$ & $594 / 3578(16.6 \%)$ \\
\hline SPMS & $142 / 723(19.6 \%)$ & $58 / 723(8.0 \%)$ & $84 / 723(11.6 \%)$ \\
\hline PPMS & $42 / 425(9.9 \%)$ & $3 / 425(0.7 \%)$ & $39 / 425(9.2 \%)$ \\
\hline
\end{tabular}

Footnote to Table 2: MS multiple sclerosis; CIS clinically isolated syndrome; RRMS relapsing remitting MS; SPMS secondary progressive MS; PPMS primary progressive MS; EDSS expanded disability status scale; F female; M male. SPMS was defined as diagnosed by the treating neurologist. Highly active disease was defined by at least 2 relapses during the previous year or at least one relapse during the previous year in spite of DMT treatment. IFN (Interferon beta), GA (Glatiramer acetate), DMF (Dimethyl fumarate) and TRF (Teriflunomide). Infusion therapies include Natalizumab, Alemtuzumab, Ocrelizumab, Mitoxantrone and Rituximab.

This article is protected by copyright. All rights reserved. 


\section{Figure legends}

Figure 1. The user interface of the Finnish MS register. First page of the user interface of the Finnish MS register gives an overview of the individual clinical history: MS diagnosis date (green dots), the diagnostic criteria used and the disease type (CIS, RRMS, SPMS or PPMS); disease modifying therapies (coloured bars); relapses (brown dots); methylprednisolone pulse therapies (violet dots); expanded disability status (EDSS) scores (blue dots and line); brain and spinal cord magnetic resonance imaging (MRI) dates, total number and location of lesions, numbers of new lesions and Gadolinium enhancing lesions; laboratory test results. The interface includes a fully automated EDSS calculator for EDSS estimation. Entering MRI data is optional. Laboratory values are obtained in most hospitals automatically by integration of the hospital laboratory database into the register.

Figure 2. Geographical presentation of MS register coverage in the Finnish health care districts. The Finnish health care districts are listed in order of population size, and the number of MS patients in the register in each district is given (10). In the map, the health care districts who have not yet joined the register are coloured grey, and the patient numbers retrieved from their discharge data are shown in the Table. The five hospital districts coloured in dark blue are analyzed in detail in this study. The health care districts using diagnosis (dg) integration and thence with coverage of all the MS patients in the administrative hospital discharge data also in the register, are shown. The number of MS patients in these hospital districts represents a crude MS prevalence at 31.12.2018 in these areas. Discharge data gave altogether 778 patients, and with the 8722 patients listed in the register, we reach a total of 9500 MS patients.

Figure 3. The age (A), time since diagnosis (B) and EDSS distribution (C) of the RRMS and SPMS patients registered in the five hospital districts with retrospective data. The five hospital districts were Helsinki and Uusimaa, Southwest Finland, Northern Savonia, Tavastia Proper, and Central Finland. There were a total of 4642 RRMS patients and 723 SPMS patients available in the register for this analysis. RR refers to RRMS and SP to SPMS. Density on the y axis refers to probability for $\mathrm{x}$-axis values. SPMS was defined as diagnosed by the treating neurologist.

This article is protected by copyright. All rights reserved. 

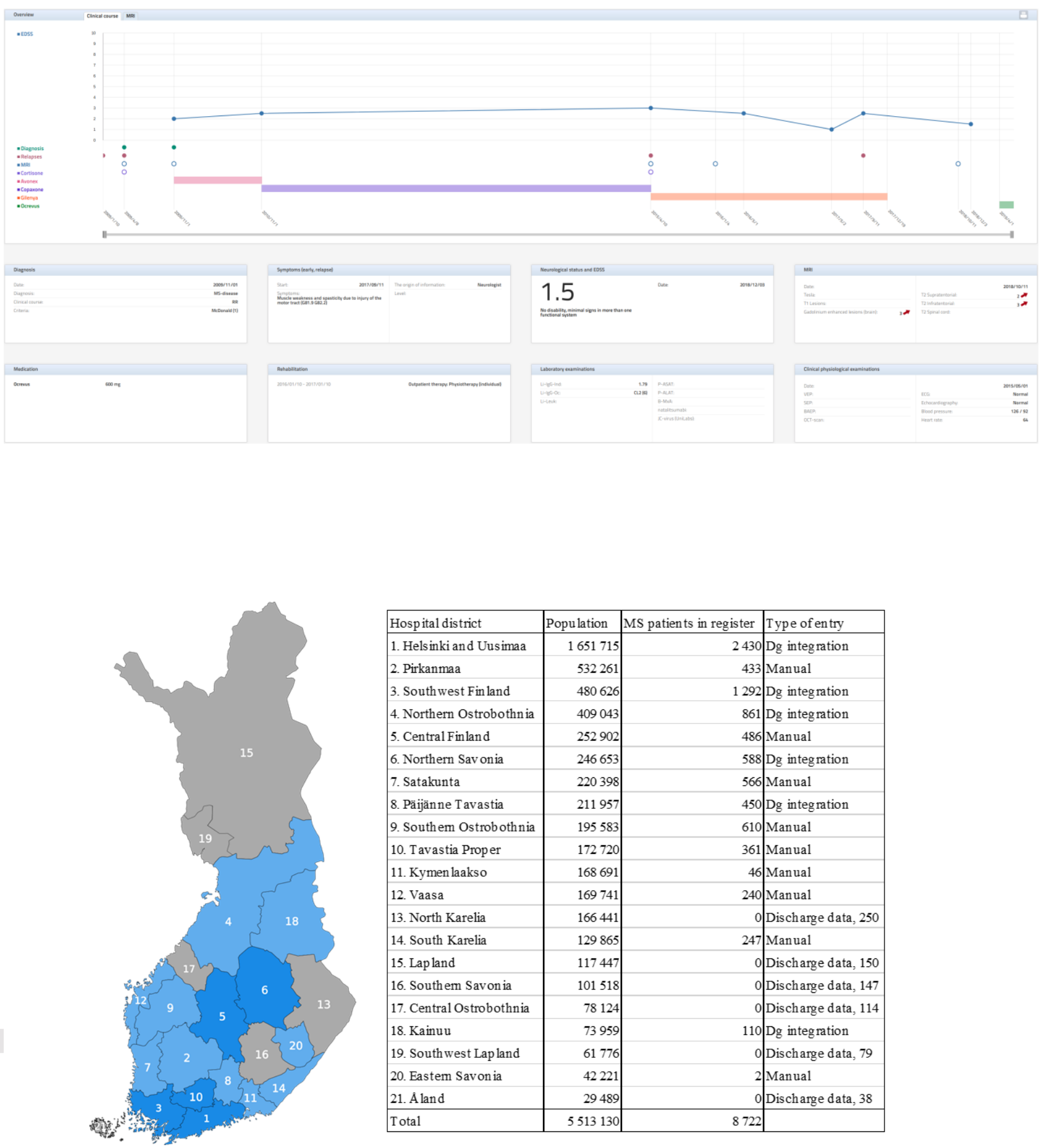

\begin{tabular}{|l|r|r|l|}
\hline Hospital district & Population & MS patients in register & Type of en try \\
\hline 1. Helsinki and Uusimaa & 1651715 & 2430 & Dg integ ration \\
\hline 2. Pirkanmaa & 532261 & 433 & Manual \\
\hline 3. Southwest Fin land & 480626 & 1292 & Dg integration \\
\hline 4. Northern Ostrobothn ia & 409043 & 861 & Dg integration \\
\hline 5. Central Finland & 252902 & 486 & Manual \\
\hline 6. Northern Sav onia & 246653 & 588 & D integration \\
\hline 7. Satakunta & 220398 & 566 & Manual \\
\hline 8. Päijänne Tavastia & 211957 & 450 & Dg integration \\
\hline 9. Southem Ostrobothnia & 195583 & 610 & Manual \\
\hline 10. Tavastia Proper & 172720 & 361 & Manual \\
\hline 11. Kymen laakso & 168691 & 46 & Manual \\
\hline 12. Vaasa & 169741 & 240 & Manual \\
\hline 13. North Karelia & 166441 & 0 & Discharge data, 250 \\
\hline 14. South Karelia & 129865 & 247 & Manual \\
\hline 15. Lapland & 117447 & 0 & ischarge data, 150 \\
\hline 16. Southem Savonia & 101518 & 0 & Discharge data, 147 \\
\hline 17. Central Ostrobothnia & 78124 & 0 & ischarge data, 114 \\
\hline 18. Kainuu & 73959 & 110 & Dg integration \\
\hline 19. Southwest Lap land & 61776 & 0 & Discharge data, 79 \\
\hline 20. Eastem Savonia & 42221 & 2 & Manual \\
\hline 21. Âland & 29489 & 0 & Discharge data, 38 \\
\hline Total & 5513130 & 8722 & \\
\hline
\end{tabular}

This article is protected by copyright. All rights reserved. 

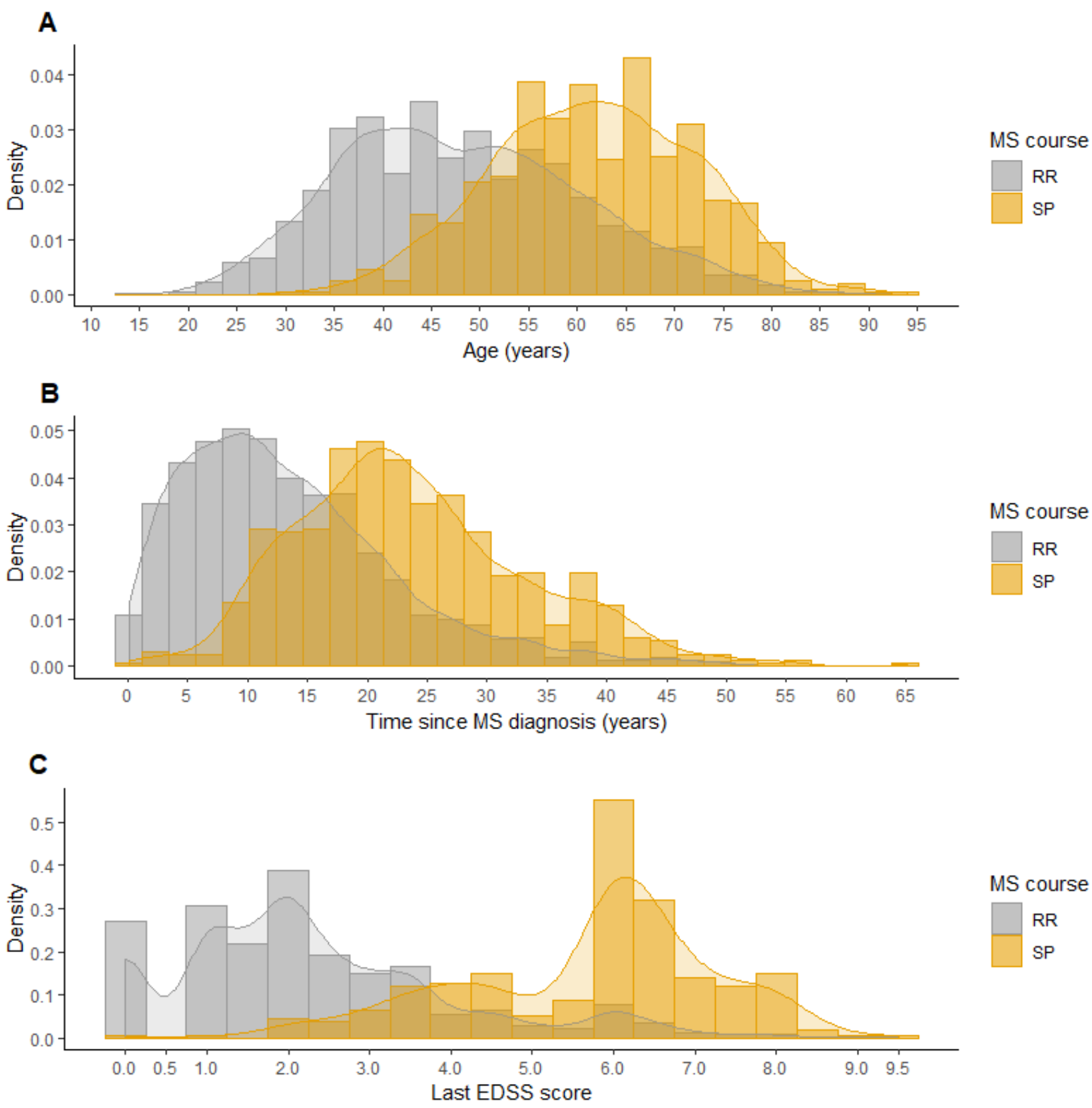

MS course

RR

SP

MS course

\begin{tabular}{|l|l|}
\hline RR \\
\hline SP
\end{tabular}

This article is protected by copyright. All rights reserved. 